International Journal of Pure and Applied Mathematics

Volume 95 No. 4 2014, 589-592

ISSN: 1311-8080 (printed version); ISSN: 1314-3395 (on-line version)

url: http://www.ijpam.eu

doi: http://dx.doi.org/10.12732/ijpam.v95i4.11

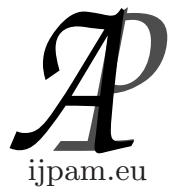

\title{
REFLEXIVE NON-DEROGATORY MATRICES
}

\author{
Y. Jahanshahi ${ }^{1}$, B. Yousefi ${ }^{2} \S$ \\ ${ }^{1,2}$ Department of Mathematics \\ Payame Noor University \\ P.O. Box: 19395-4697, Tehran, IRAN
}

\begin{abstract}
A matrix $A$ is called reflexive if and only if $\operatorname{Lat}(A) \subseteq \operatorname{Lat}(B)$ implies that $B=p(A)$ for some polynomial $p$. In this article, we characterize reflexive non-derogatory matrices.
\end{abstract}

Key Words: invariant subspace, reflexive matrix, Jordan block, non-derogatory matrix

\section{Introduction}

Let $M_{n}$ be the algebra all $n \times n$ complex matrices. For any matrix $A \in M_{n}$, a closed linear subspace $F$ of $\mathbb{C}^{n}$ is $A$-invariant if $A(F) \subseteq F$. We let $\operatorname{Lat}(A)$ denote the lattice of all closed subspaces invariant for $A$, and $A \lg \operatorname{Lat}(A)$ is the algebra of all matrices $B \in M_{n}$ such that $\operatorname{Lat}(A) \subseteq \operatorname{Lat}(B)$. A matrix $A \in M_{n}$ is said to be reflexive if $\operatorname{Alg} \operatorname{Lat}(A)=W(A)$, where $W(A)$ is the weakly closed algebra generated by $A$ and $I$. Actually, $A \in M_{n}$ is reflexive if and only if $\operatorname{Lat}(A) \subset \operatorname{Lat}(B)$ implies that $B=p(A)$ for some polynomial $P$.

Let $A \in M_{n}$. If $m=\prod_{i=1}^{n} p_{i}^{r_{i}}$ is the minimal polynomial of $A$, with

Received: June 14, 2014

(C) 2014 Academic Publications, Ltd. url: www.acadpubl.eu

${ }^{\S}$ Correspondence author 
$p_{1}, p_{2}, \ldots, p_{n}$ distinct irreducible monic polynomials, we recall that the subspaces $M_{i}=\operatorname{kerp}_{i}(A)^{r_{i}} ; 1 \leq i \leq n$, are invariant for $A$, are linearly independent, and span $\mathbb{C}^{n}$. Suppose that $A_{i}=\left.A\right|_{M_{i}}$ for $i=1, \ldots, n$. In [1] Deddens and Fillmore showed that if $A=\sum_{i=1}^{n} \oplus A_{i}$, then $A$ is reflexive if and only if each $A_{i}$ is reflexive. A square complex matrix $A \in M_{n}$ with eigenvalues $\lambda_{1}, \lambda_{2}, \ldots, \lambda_{n}$ is similar to a block diagonal matrix

$$
J=\left(\begin{array}{cccc}
J_{1}\left(\lambda_{1}\right) & 0 & \cdots & 0 \\
0 & J_{2}\left(\lambda_{2}\right) & 0 & \vdots \\
\vdots & \cdots & \ddots & 0 \\
0 & \cdots & 0 & J_{k}\left(\lambda_{k}\right)
\end{array}\right)
$$

where $1 \leq k \leq n$ and each block $J_{i}\left(\lambda_{i}\right)$ is a square matrix of the form

$$
J_{i}\left(\lambda_{i}\right)=\left(\begin{array}{cccc}
\lambda_{i} & 1 & & 0 \\
0 & \lambda_{i} & \ddots & \\
& & \ddots & 1 \\
0 & & 0 & \lambda_{i}
\end{array}\right) .
$$

So there exists an invertible matrix $P$ such that $P^{-1} A P=J$ is such that the only non-zero entries of $J$ are on the diagonal and the superdiagonal. The matrix $J$ is called the Jordan normal form of $A$. Each $J_{k}(\lambda)$ is called a Jordan block of $A$. A matrix $A$ is non-derogatory if and only if it's characteristic polynomial coincides with it's minimum polynomial. For some sources on these topics one can see [1-6].

\section{Reflexivity of Non-Derogatory Matrices}

For the proof of the main result we will need the following lemma.

Lemma 2.1. Let $J_{k}(\lambda)$ be a $k \times k$ Jordan block. $\operatorname{Lat}\left(J_{k}(\lambda)\right)$ is

$$
E=\left\{0, \operatorname{span}\left(e_{1}\right), \operatorname{span}\left(e_{1}, e_{2}\right), \ldots, \operatorname{span}\left(e_{1}, e_{2}, \ldots, e_{k-1}\right), \mathbb{C}^{k}\right\} .
$$

Proof. It is easy to show that $E$ is $J_{k}(\lambda)$-invariant. It is sufficient that, if $W$ is a $J_{k}(\lambda)$-invariant subspace of $\mathbb{C}^{k}$ of dimension at least 1 , then $W \in E$. Let $J_{k}(\lambda) W \subseteq W$, since $e_{1}$ is the only eigenvector of $J_{k}(\lambda)$, so $e_{1} \in W$. Suppose 
$e_{2} \notin W$. For every $w \in W$, we have $w=\left(w_{1}, 0, w_{2}, \ldots, w_{k}\right)^{T}$. Hence $J_{k}(\lambda) W \subseteq$ $W$ which implies that

$$
\left(\begin{array}{cccc}
\lambda & 1 & & 0 \\
0 & \lambda & \ddots & \\
& & \ddots & 1 \\
0 & & 0 & \lambda
\end{array}\right)\left(\begin{array}{c}
w_{1} \\
0 \\
\vdots \\
w_{k}
\end{array}\right)=\left(\begin{array}{c}
\lambda w_{1} \\
w_{3} \\
\vdots \\
w_{k}
\end{array}\right)=\left(\begin{array}{c}
w_{1}^{\prime} \\
0 \\
\vdots \\
w_{k}^{\prime}
\end{array}\right)
$$

where $w^{\prime}=\left(\begin{array}{lllll}w_{1}^{\prime} & 0 & w_{2}^{\prime} & \cdots & w_{k}^{\prime}\end{array}\right)^{T} \in W$. So $w_{3}=0$ and $e_{3} \notin W$. It follows that if $e_{i} \notin W$ for $i=2,3, \ldots, k-1$, then $e_{i+1} \notin W$. Hence $W \in E$.

Theorem 2.2. Every Jordan block of degree at least 2 is not reflexive.

Proof. Let $B=(B)_{i, j} \in M_{n}$ and $\operatorname{Lat}\left(J_{n}(\lambda)\right) \subseteq \operatorname{Lat}(B)$. So

$$
E=\left\{0, \operatorname{span}\left(e_{1}\right), \operatorname{span}\left(e_{1}, e_{2}\right), \ldots, \operatorname{span}\left(e_{1}, e_{2}, \ldots, e_{k-1}\right), \mathbb{C}^{k}\right\} \subseteq \operatorname{Lat}(B) .
$$

Since $e_{1} \in E$, we have

$$
\left(\begin{array}{cccc}
b_{1,1} & b_{1,2} & \cdots & b_{1, n} \\
b_{2,1} & b_{2,2} & \cdots & b_{2, n} \\
\vdots & \vdots & & \vdots \\
b_{n, 1} & b_{n, 2} & \cdots & b_{n, n}
\end{array}\right)\left(\begin{array}{c}
1 \\
0 \\
\vdots \\
0
\end{array}\right)=\left(\begin{array}{c}
b_{1,1} \\
b_{2,1} \\
\vdots \\
b_{n, 1}
\end{array}\right)
$$

which implies that $b_{2,1}=b_{3,1}=\cdots=b_{n, 1}=0$, and $\left(\begin{array}{c}1 \\ 1 \\ 0 \\ \vdots\end{array}\right) \in E$. Hence

$$
\left(\begin{array}{cccc}
b_{1,1} & b_{1,2} & \cdots & b_{1, n} \\
0 & b_{2,2} & \cdots & b_{2, n} \\
\vdots & \vdots & & \vdots \\
0 & b_{n, 2} & \cdots & b_{n, n}
\end{array}\right)\left(\begin{array}{c}
1 \\
1 \\
0 \\
\vdots
\end{array}\right)=\left(\begin{array}{c}
b_{1,1}+b_{1,2} \\
b_{2,2} \\
\vdots \\
b_{2, n}
\end{array}\right)
$$

and so $b_{3,2}=b_{4,2}=\cdots=b_{n, 2}=0$. Consequently, $B$ should be an upper triangular matrix. In the other hand, $B=p\left(J_{n}(\lambda)\right)$ implies that

$$
B=c_{0} I+c_{1} J_{n}(\lambda)+\ldots+c_{n}\left(J_{n}(\lambda)\right)^{n},
$$

where $c_{i}$ for $i=1,2, \ldots, n$ are constant complex numbers. 
Hence

$$
\begin{aligned}
b_{11} & =c_{0}+c_{1} \lambda+\ldots+c_{n} \lambda^{n}, \\
b_{22} & =c_{0}+c_{1} \lambda+\ldots+c_{n} \lambda^{n}, \\
& \vdots \\
b_{n n} & =c_{0}+c_{1} \lambda+\ldots+c_{n} \lambda^{n} .
\end{aligned}
$$

It follows that all entries on diagonal of $B$ are equal. So by the above discussion $J_{n}(\lambda)$ is not reflexive.

Corollary 2.3. Let $A$ be a non-derogatory matrix. Then $A$ is reflexive if and only if every eigenvalue of $A$ has algebraic multiplicity of 1 .

Proof. By using Theorem 1 in [1] and Theorem 2.2, the proof is complete.

\section{References}

[1] J. A. Deddens and P. A. Fillmore, Reflexive linear transformation, Linear Algebra and Appl., 10 (1975), 89-93.

[2] R. Horn and C. Johnson, Matrix Analysis, Cambridge University Press, London (1985).

[3] S. Monica Osnaga, On rank one matrices and invariant subspaces, Balkan Journal of Geometry and its Appl., 10 (2005), 383-406.

[4] H. Radjavi and P. Rosenthal, Invariant Subspaces, Eng. Der Math. Und ihrer Grenzbegiete, Bd. 77, Springer, Berlin (1973).

[5] K. Seddighi and B. Yousefi, On the reflexivity of operators on function spaces, Proc. Amer. Math. Soc., 116 (1992), 45-52.

[6] B. Yousefi, Multiplication operators on Hilbert spaces of analytic functions, Archiv der Mathematik, 83, No. 6 (2004), 536-539. 